\title{
Radiative background to the search for right-handed charged currents *
}

\author{
H.F. Contopanagos ** and M.B. Einhorn $* * *$ \\ Randall Laboratory of Physics, University of Michigan, Ann Arbor, MI 48109-1120, USA \\ and \\ Centre de Physique Théorique, CNRS Luminy - Case 907, F-13288 Marseille Cedex 9, France
}

Received 27 June 1989

(Revised 1 November 1991)

Accepted for publication 27 December 1991

\begin{abstract}
The probability for an electron to emit a collinear, hard photon while undergoing helicity flip is finite in the limit that the electron mass tends to zero. We point out that this provides a background of order $\alpha$ to tests of the Standard Model, which may affect the search for right-handed, charged currents in deeply inelastic scattering, especially at HERA. It would appear to be exceedingly difficult to discriminate against this background in practice and may make it difficult to improve substantially the mass limits on a right-handed charged vector boson.
\end{abstract}

\section{Introduction}

One of the exciting features that HERA is expected to have is the capacity to produce longitudinally polarized electron and positron beams [1,2]. One application of this capability will be to search for right-handed charged currents, which are completely absent in the Standard Model (SM), via $\mathrm{e}_{\mathrm{R}}^{-} \mathrm{p} \rightarrow \nu_{\mathrm{e}} X$. (See fig. 1.) The SM cross section $\sigma_{\mathrm{L}}^{\mathrm{R}}$ for a right-helicity electron $\mathrm{e}_{\mathrm{R}}^{-}$of energy $E_{\mathrm{c}}$ to scatter via the usual $\mathrm{W}^{-}$exchange is suppressed relative to the usual cross section $\sigma_{\mathrm{L}}^{\mathrm{L}}$ for a left-helicity electron by a factor of $\left(m_{\mathrm{e}} / E_{\mathrm{e}}\right)^{2}$, where $m_{\mathrm{e}}$ is the electron mass *. For a $26 \mathrm{GeV}$ electron, this is quite negligible $\left(\simeq 4 \times 10^{-10}\right.$.)

In this note, we would like to point out a surprising background which may limit the ability to perform such a search. This results from bremsstrahlung by $e_{R}^{-}$of a

* Work supported in part by the Department of Energy of the US and the CNRS of France.

* * Present address: Institute for Theoretical Physics, State University of New York, Stony Brook, NY 11794-3840, USA.

*** Address until Aug., 1992: Institute for Theoretical Physics, University of California, Santa Barbara, CA 93106-4030, USA.

* Our notation for the cross section for interaction of an electron of helicity $\lambda$ with a left-handed (right-handed) current will be $\sigma_{\mathrm{L}}^{\lambda}\left(\sigma_{\mathrm{R}}^{\lambda}\right)$. 


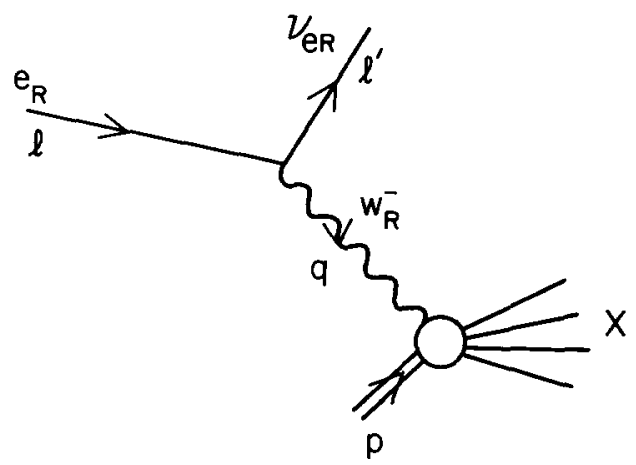

Fig. 1. Deeply inelastic scattering from a proton by a right-helicity electron to a right-helicity neutrino via exchange of a hypothetical $W_{R}^{-}$vector boson.

forward-going photon accompanied by a helicity flip, enabling the electron to participate in the usual left-handed, charged current interaction (fig. 2). * Since the final state neutrino $\nu_{\mathrm{eL}}$ goes undetected and the emitted photon simply proceeds unobserved down the beam pipe, it is not possible to discriminate this from the process of interest (fig. 1). One might think that, because this involves electron helicity flip, the ratio of this to the usual deeply inelastic scattering cross section for a left-helicity electron would be proportional to $\alpha\left(m_{\mathrm{e}} / E_{\mathrm{e}}\right)^{2}$, where $\alpha \equiv e^{2} / 4 \pi$. However, as $m_{\mathrm{e}} \rightarrow 0$, the probability of helicity flip tends to a finite, non-zero limit because of a collinear singularity in the electron propagator, a circumstance noted long ago by Lee and Nauenberg in their classic paper on mass singularities [3]. Thus, the cross section $\sigma_{\mathrm{L}}^{\mathrm{R}, \gamma}$ is only down by a factor of $\alpha$. Inasmuch as the cross section for a heavy vector boson falls as $1 / M_{\mathrm{R}}^{4}$, one might expect $\sigma_{\mathbf{L}}^{\mathrm{R}, \gamma}$ to be roughly of the same order as a right-handed vector boson in the

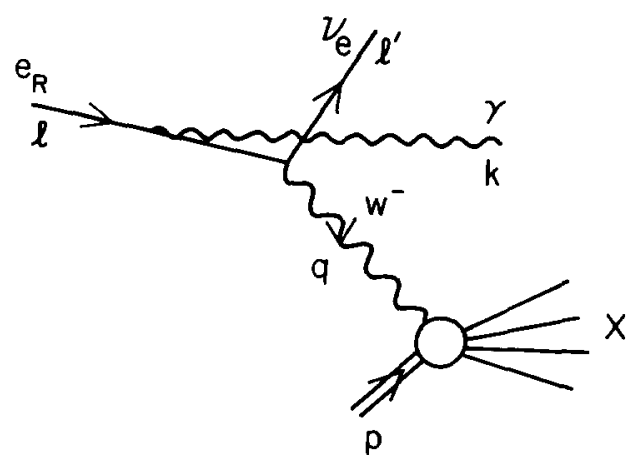

Fig. 2. Radiative deeply inelastic scattering from a proton of a right-helicity electron via a Standard Model $\mathrm{W}^{-}$exchange.

* The cross section for this will be denoted as $\sigma_{\mathrm{L}}^{\mathrm{R}, \gamma}$. 
mass range to be probed at HERA. It is important to take this into account, since otherwise such experiments at HERA might be incorrectly interpreted as having provided evidence for right-handed charged currents.

An outline of this paper is as follows: In sect. 2, we review the cross section for a right-handed charged current, and in sect. 3, we determine the cross section for the radiative background described above and describe the implications this background would have for experiments. Finally, in sect. 4, we consider briefly other potential applications and summarize our conclusions.

\section{Right-handed charged current}

Before taking up the radiative process, let us first record the cross section for a right-handed vector boson. (Fig. 1.) The standard definitions of kinematical variables are

$$
s \equiv(p+l)^{2}, \quad q^{2} \equiv\left(l-l^{\prime}\right)^{2} \equiv-Q^{2}, \quad W^{2} \equiv(q+p)^{2},
$$

or scaling variables

$$
x \equiv \frac{Q^{2}}{2 q \cdot p}, \quad y \equiv \frac{p \cdot q}{p \cdot l} .
$$

Note that $Q^{2}=x y s$ and $W^{2}=(1-x) y s$. With much of the proton's fragments contained within the beam pipe and the right-handed $\nu_{\mathrm{eR}}$ going undetected ${ }^{\star}$, the only final state observable is the current jet's momentum $p_{\mathrm{J}} \equiv x p+q$ formed by fragmentation of the outgoing quark. Thus, the kinematical variables $x, y$ must be inferred from $p_{\mathrm{J}} \cdot p$ and $p_{\mathrm{J}} \cdot l$, by noting that

$$
\frac{p_{\mathrm{J}} \cdot p}{p \cdot l}=\frac{E_{\mathrm{J}}}{E_{\mathrm{e}}} \sin ^{2} \frac{\theta_{\mathrm{J}}}{2} \approx y, \quad \frac{p_{\mathrm{J}} \cdot l}{p \cdot l}=\frac{E_{\mathrm{J}}}{E_{\mathrm{p}}} \cos ^{2} \frac{\theta_{\mathrm{J}}}{2}=x(1-y),
$$

where $E_{\mathrm{J}}, \theta_{\mathrm{J}}$ denote the energy and production angle, respectively, with $\theta_{\mathrm{J}}$ measured relative to the proton beam. As is customary in quoting limits on the mass $M_{\mathrm{R}}$ of a vector boson $\mathrm{W}_{\mathrm{R}}^{-}$coupling to fermions as $\mathrm{V}+\mathrm{A}$, we will assume its coupling has the same strength $\left(g_{2}=e / \sin \theta_{\mathrm{w}}\right)$ as the usual $\mathrm{V}-\mathrm{A} \mathrm{SM} \mathrm{W} \mathrm{W}^{-}$. Then the cross section may be written as

$$
\frac{\mathrm{d} \sigma_{\mathrm{R}}^{\mathrm{R}}}{\mathrm{d} x \mathrm{~d} y}=\frac{\pi \alpha_{2}^{2}}{4 s\left(x y+M_{\mathrm{R}}^{2} / s\right)^{2}}\left[x y^{2} G_{1}+(1-y) G_{2}+x y\left(1-\frac{y}{2}\right) G_{3}\right],
$$

\footnotetext{
* In some models, some information might be inferred from its decay products.
} 
where $\alpha_{2} \equiv g_{2}^{2} / 4 \pi$ and $M_{\mathrm{R}}$ is the mass of $\mathrm{W}_{\mathrm{R}}$ and the $G_{i}=G_{i}\left(x, Q^{2}\right)(i=1,2,3)$ are the usual dimensionless structure functions *. For large $\mathrm{Q}^{2}$, these scale (up to QCD corrections) with

$$
G_{2}=2 x G_{1}=2 x(U(x)+\bar{D}(x)), \quad G_{3}=2(U(x)-\bar{D}(x)),
$$

where $U(\bar{D})$ denotes the sum of contributions from quarks (antiquarks) of charge $+\frac{2}{3}\left(+\frac{1}{3}\right.$.) This gives

$$
\frac{\mathrm{d} \sigma_{\mathrm{R}}^{\mathrm{R}}}{\mathrm{d} x \mathrm{~d} y}=\frac{\underline{\pi \alpha_{2}^{2}}}{2 s\left(x y+M_{\mathrm{R}}^{2} / s\right)^{2}} x\left[U(x)+(1-y)^{2} \bar{D}(x)\right],
$$

and we may estimate the integral over $y$ by neglecting the $Q^{2}$ evolution of $U$ and $\bar{D}$, giving

$$
\begin{aligned}
\frac{\mathrm{d} \sigma_{\mathrm{R}}^{\mathrm{R}}}{\mathrm{d} x}= & \frac{\pi \alpha_{2}^{2}}{2 M_{\mathrm{R}}^{2}}\left[\left(1+\frac{1}{x \zeta_{\mathrm{R}}}\right)^{-1} U(x)\right. \\
& \left.+\left(1+\frac{2}{x \zeta_{\mathrm{R}}}-\frac{2}{x \zeta_{\mathrm{R}}}\left(1+\frac{1}{x \zeta_{\mathrm{R}}}\right) \ln \left(1+x \zeta_{\mathrm{R}}\right)\right) \bar{D}(x)\right],
\end{aligned}
$$

where we defined $\zeta_{\mathrm{R}}=s / M_{\mathrm{R}}^{2}$. For small $x\left(x \zeta_{\mathrm{R}} \ll 1\right)$, this behaves as

$$
\frac{\mathrm{d} \sigma_{\mathrm{R}}^{\mathrm{R}}}{\mathrm{d} x}=\frac{\pi \alpha_{2}^{2} S}{4 M_{\mathrm{R}}^{4}} x\left[U(x)+\frac{1}{3} \bar{D}(x)\right]
$$

Given the quark distribution functions, an upper limit on $\sigma_{\mathrm{R}}^{\mathrm{R}}$ may be interpreted as a lower limit on $M_{\mathrm{R}}$. Present limits on $M_{\mathrm{R}}$ stem from muon beta decay, non-leptonic weak decays, and the $K_{L}-K_{S}$ mass difference [4]. The limits are process- and model-dependent, ranging from about $300 \mathrm{GeV}$ to several TeV. Models differ in the expectations as to whether the produced neutrino is Majorana or Dirac, massive or massless. The largest cross sections generally are associated with a light, Dirac neutrino, so this is the case we shall analyze in detail. Estimates have suggested that, in the case of a light, Dirac $\nu_{\mathrm{eR}}$, experiments at HERA might be sensitive to $M_{\mathrm{R}}$ as large as $300-500 \mathrm{GeV}$, depending on the luminosity anticipated [5-7]. The question we wish to address is the degree to which the bremsstrahlung process depicted in fig. 2 competes.

* These are also called $m_{\mathrm{p}} W_{1}, \nu W_{2}$ and $\nu W_{3}$, respectively. 


\section{3. $\mathrm{e}_{\mathrm{R}}^{-} \mathrm{p} \rightarrow v_{\mathrm{e}} \gamma X$}

Before proceeding with the detailed calculation, let's do a "back-of-the-envelope" estimate. As we shall see, the total probability of helicity flip bremsstrahlung is $\alpha / 4 \pi$, so we would guess that the ratio $\left(\mathrm{d} \sigma_{\mathrm{R}}^{\mathrm{R}} / \mathrm{d} Q^{2}\right) /\left(\mathrm{d} \sigma_{\mathrm{L}}^{\mathrm{R}, \gamma} /\right.$ $\mathrm{d} Q^{2}$ ) would be of order

$$
\frac{\alpha}{4 \pi} \frac{\left(Q^{2} \pm M_{\mathrm{R}}^{2}\right)^{2}}{\left(Q^{2}+M_{\mathrm{W}}^{2}\right)^{2}} .
$$

For $Q^{2} \ll M_{\mathrm{W}}^{2}$, we find equality for $M_{\mathrm{R}} \simeq 515 \mathrm{GeV}$, somewhat lower than the more precise results below ( $\simeq 680 \mathrm{GeV}$.) To maximize the sensitivity to $M_{\mathrm{R}}$, one wants to make a cut in $Q^{2}$ as large as possible consistent with having an adequate number of events to minimize statistical errors. It appears that, given the design luminosity, one might reasonably choose $[5,6] Q^{2} \geqslant 5000 \mathrm{GeV}^{2}$. For that value of $Q^{2}$, the ratio in eq. (3.1) is 1 for $M_{\mathrm{R}} \approx 685 \mathrm{GeV}$, in good agreement with more accurate calculation $(\simeq 780 \mathrm{GeV}$.) Beyond this mass, the bremsstrahlung process will dominate, yielding an unavoidable background shielding any potential righthanded current events. So the cut in $Q^{2}$ would be important to reach the anticipated limits of HERA's sensitivity and certainly crucial for going beyond this.

The calculation of $\sigma_{\mathrm{L}}^{\mathrm{R}, \gamma}$ is straightforward but kinematically more complicated than the non-radiative process. Of the several diagrams contributing, only the one depicted in fig. 2 has a mass singularity leading to a finite rate in the limit $m_{\mathrm{e}} \rightarrow 0$. (While not gauge invariant by itself, in the limit $m_{\mathrm{e}} \rightarrow 0$ it is gauge invariant.) With the kinematics as depicted in fig. 2 , the differential cross section may be written as

$$
\begin{aligned}
\mathrm{d} \sigma_{\mathrm{L}}^{\mathrm{R}, \gamma}= & \frac{\underline{\alpha} \underline{\alpha}_{2}^{2}}{16 \pi^{2}} \int \mathrm{d}^{4} q \int\left\{\frac{\underline{k_{0}^{2} \mathrm{~d} \Omega_{\gamma}}}{k \cdot(l-q)}\right\} \frac{\underline{m}_{e}^{2}}{(k \cdot l)^{2}} \\
& \times\left(\frac{p \cdot k}{p \cdot l}\right)^{2} \frac{1}{y^{\prime}}\left[x y^{\prime 2} G_{1}+\left(1-y^{\prime}\right) G_{2}+x y^{\prime}\left(1-\frac{y^{\prime}}{2}\right) G_{3}\right],
\end{aligned}
$$

where the angular integration is over the direction of the radiated photon. Here we have introduced another scaling variable

$$
y^{\prime} \equiv \frac{p \cdot q}{p \cdot(l-k)},
$$

which evidently plays the same role for the radiative process as does $y$ in the non-radiative case. It is restricted to the range

$$
y \leqslant y^{\prime} \leqslant 1,
$$

with the lower limit corresponding to a zero-momentum photon. 
For a given momentum transfer $q$, the photon energy $k_{0}$ and direction are related by the requirement that $l^{\prime 2}=(l-k-q)^{2}=m_{\nu_{\mathrm{CR}}}^{2}$, which we have assumed to be small *. In the limit $m_{\mathrm{e}} \rightarrow 0$, this would appear to vanish, but, because $k \cdot l$ becomes singular in the forward direction in this limit, one obtains a finite result. Integrating the photon direction over a small cone (angle $\Delta \theta$ ) about the electron direction $l$, we have

$$
k_{0}^{2} \frac{m_{\mathrm{e}}^{2}}{4 \pi} \int \frac{\mathrm{d} \Omega_{\gamma}}{(k \cdot l)^{2}}=\frac{m_{\mathrm{e}}^{2}}{E_{\mathrm{e}}^{2}} \int_{0}^{\Delta \theta} \frac{\mathrm{d} \theta^{2}}{\left(\theta^{2}+\left(m_{\mathrm{e}}^{2} / E_{\mathrm{e}}^{2}\right)\right)^{2}}=\frac{\Delta \theta^{2}}{\Delta \theta^{2}+\left(m_{\mathrm{e}}^{2} / E_{\mathrm{e}}^{2}\right)} .
$$

Thus, for $m_{\mathrm{e}} / E_{\mathrm{e}} \ll \Delta \theta$, this is finite, approximately independent of $\Delta \theta$ [3]. Since at HERA, $E_{\mathrm{e}} \approx 26 \mathrm{GeV}$, one would have to resolve the photon within an angle $\Delta \theta \leqq 10^{-5}$. Within the detector region, this photon therefore not only is inside the beam pipe but is within the electron beam itself! Thus the photon proceeds forward unobserved, carrying away an unknown amount of energy.

Before continuing with the experimental implications, we could like to indicate that this can be transformed into a much more intuitive and easily interpretable form. It is convenient to define yet another scaling variable

$$
z^{\prime} \equiv \frac{p \cdot(l-k)}{p \cdot l}
$$

corresponding to the longitudinal momentum fraction carried by the intermediate, left-helicity electron which, in the limit $m_{\mathrm{e}} \rightarrow 0$, is on the mass shell. (Note that $z^{\prime}=y / y^{\prime}$.) Then, after some labor, eq. (3.2) can be brought into the form

$$
\frac{\frac{\mathrm{d} \sigma_{\perp}^{\mathrm{R}, \gamma}}{\mathrm{d} x \mathrm{~d} y^{\prime} \mathrm{d} z^{\prime}}}{z^{\prime}} P_{-+}\left(z^{\prime}\right) \frac{\frac{\mathrm{d} \sigma_{\perp}^{\mathrm{L}}}{\mathrm{d} x \mathrm{~d} y^{\prime}}}{,}
$$

where

$$
P_{-+}\left(z^{\prime}\right) \equiv \frac{\alpha}{2 \pi} z^{\prime}\left(1-z^{\prime}\right)
$$

and

$$
\frac{\mathrm{d} \sigma_{L}^{\mathrm{L}}}{\mathrm{d} x \mathrm{~d} y^{\prime}}=\frac{\pi \alpha_{2}^{2}}{4 s^{\prime}\left(x y^{\prime}+M_{\mathrm{W}}^{2} / s^{\prime}\right)}\left[x y^{\prime 2} G_{1}+\left(1-y^{\prime}\right) G_{2}+x y^{\prime}\left(1-\frac{y^{\prime}}{2}\right) G_{3}\right] .
$$

$P_{-+}\left(z^{\prime}\right)$ is the probability to find a left-chirality electron of momentum $z^{\prime}$ in a right-helicity electron, while $\left(\mathrm{d} \sigma_{\mathrm{L}}^{\mathrm{L}} / \mathrm{d} x \mathrm{~d} y^{\prime}\right)$ denotes the usual SM charged-current

\footnotetext{
* Despite appearances, the factor inside the curly bracket is Lorentz invariant.
} 
cross section at the degraded energy $s^{\prime} \equiv z^{\prime} s$. Note that $P_{-+}\left(z^{\prime}\right)$ arises from a collinear mass-shell singularity. In this respect, it resembles the Altarelli-Parisi [8] "splitting functions," but it is a long-distance rather than a short-distance effect which does not contribute to the evolution equations, the transverse momentum integration being convergent. It is more analogous to the quark distributions in a proton than to the splitting functions $P_{A B}$. It would itself evolve according to the Altarelli-Parisi equations adapted to QED rather than QCD. Note that the total probability of finding a left-helicity electron in a right-helicity electron is

$$
\int_{0}^{1} \frac{\mathrm{d} z^{\prime}}{z^{\prime}} P_{-+}\left(z^{\prime}\right)=\frac{\alpha}{4 \pi},
$$

a fact we used earlier in making our "back-of-the-envelope" estimate.

Returning to experimental issues and noting once again that the only final state variable which may be experimentally determined is the quark jet momentum $p_{\mathrm{J}}$, then the only observables are $y$ and

$$
z_{\mathrm{J}} \equiv \frac{p_{\mathrm{J}} \cdot l}{p \cdot l},
$$

(See eq. (2.1).) However, unlike the non-radiative case, the relation $z_{\mathbf{J}}=x(1-y)$ does not obtain, rather, $z_{\mathrm{J}}=x\left(1-y^{\prime}\right)$. In particular, the momentum transfer $q$ cannot be determined. Thus, only $y$ and $z_{\mathrm{j}}$ may be experimentally determined, with the third variable, either $x$ or $y^{\prime}$ undetermined. The triply differential cross section for this "collinear, hard emission" may be written as

$$
\begin{aligned}
\frac{\mathrm{d} \sigma_{\perp}^{\mathrm{R}, \gamma}}{\mathrm{d} x \mathrm{~d} y \mathrm{~d} z_{\mathrm{J}}}= & \frac{\alpha \alpha_{2}^{2}}{8 s} \frac{y}{\left(x y+M_{\mathrm{W}}^{2} / s\right)^{2}} \frac{\left(x(1-y)-z_{\mathrm{J}}\right)}{\left(x-z_{\mathrm{J}}\right)^{2}} \\
& \times\left[\left(x-z_{\mathrm{J}}\right) G_{1}+\left(\frac{z_{\mathrm{J}}}{x-z_{\mathrm{J}}}\right) G_{2}+\left(\frac{x+z_{\mathrm{J}}}{2}\right) G_{3}\right] .
\end{aligned}
$$

Since $x$ is not observable, the best way of interpreting data might be simply to present the experimental value of $\mathrm{d} \sigma / \mathrm{d} y \mathrm{~d} z_{\mathrm{J}}$ and not assume that $z_{\mathrm{J}}=x(1-y)$. However, because the structure functions will be well-determined by the time this test is carried out, $\mathrm{d} \sigma_{\mathrm{L}}^{\mathrm{R}, \gamma}$ may be accurately calculated and compared with the observed rate. To get a feeling for how much this background would affect results, and in order to compare directly with eqs. (2.3)-(2.6), we have calculated instead

$$
\begin{aligned}
\frac{\underline{\mathrm{d} \sigma_{\perp}^{\mathrm{R}, \gamma}}}{\mathrm{d} x \mathrm{~d} y}= & \frac{\alpha \alpha_{2}^{2}}{8 s} \frac{1}{\left(x y+M_{\mathrm{W}}^{2} / s\right)^{2}}\left[(1-y+y \ln y) x y G_{1}+\left(\frac{1}{2}\left(1-y^{2}\right)+y \ln y\right) G_{2}\right. \\
& \left.-\frac{1}{2} x y(3(1-y)+(2+y) \ln y) G_{3}\right] .
\end{aligned}
$$


Inputting the quark-parton model relations, eq. (2.4), this becomes simply

$$
\begin{aligned}
\frac{\mathrm{d} \sigma_{\mathrm{L}}^{\mathrm{R}, \gamma}}{\mathrm{d} x \mathrm{~d} y}= & \frac{\alpha \alpha_{2}^{2}}{8 s} \frac{x}{\left(x y+M_{\mathrm{W}}^{2} / s\right)^{2}} \\
& \times\left[(1-y)^{2} U(x)+((1-y)(1+5 y)+2 y(2+y) \ln y) \bar{D}(x)\right] .
\end{aligned}
$$

(The coefficient of $\bar{D}$ tends to $(1-y)^{4} / 6$ as $y \rightarrow 1$.) Integrating over $y$, we find

$$
\begin{aligned}
\frac{\mathrm{d} \sigma_{\perp}^{\mathrm{R}, \gamma}}{\mathrm{d} x}= & \frac{\alpha \alpha_{2}^{2}}{4 x s}\left[\left(\frac{x \zeta_{\mathrm{W}}}{2}+1-\left(1+\frac{1}{x \zeta_{\mathrm{W}}}\right) \ln \left(1+x \zeta_{\mathrm{W}}\right)\right) U(x)\right. \\
& +\left(\frac{x \zeta_{\mathrm{W}}}{2}-6+4\left(1+\frac{1}{x \zeta_{\mathrm{W}}}\right) \ln \left(1+x \zeta_{\mathrm{W}}\right)\right. \\
& \left.\left.+2\left(1-\frac{1}{x \zeta_{\mathrm{W}}}\right) \operatorname{Li}_{2}\left(-x \zeta_{\mathrm{W}}\right)\right) \bar{D}(x)\right]
\end{aligned}
$$

where $\operatorname{Li}_{2}(z)$ is the Spence function or dilogarithm [9] and $\zeta_{\mathrm{W}}=s / M_{\mathrm{W}}^{2}$. For small $x\left(x \zeta_{\mathrm{W}} \ll 1\right)$, this behaves as

$$
\frac{\mathrm{d} \sigma_{\mathrm{L}}^{\mathrm{R}, \gamma}}{\mathrm{d} x}=\frac{\alpha \alpha_{2}^{2}}{24} \frac{s}{M_{\mathrm{W}}^{4}} x\left[U(x)+\frac{1}{3} \bar{D}(x)\right] .
$$

In fig. 3, we compare this background with the size of the cross section for a right-handed vector boson $\mathrm{W}_{\mathrm{R}}$. The contribution of the $\bar{D}$ quarks is kinematically suppressed in $\mathrm{d} \sigma / \mathrm{d} x$ so that the ratio $\left(\mathrm{d} \sigma_{\mathrm{R}}^{\mathrm{R}} / \mathrm{d} x\right) /\left(\mathrm{d} \sigma_{\mathrm{L}}^{\mathrm{R}, \gamma} / \mathrm{d} x\right)$ is insensitive to the quark distributions. For simplicity, we have chosen the simple parameterization of the structure functions by Buras and Gaemers [10] and neglected their $Q^{2}$ variation in the integration over $y$. (We set $Q^{2}=100 \mathrm{GeV}^{2}$.) From this figure, we see that, for $x=0.01$, the background dominates over the signal for $M_{\mathrm{R}} \geq 680$ $\mathrm{GeV}$, but already for $M_{\mathrm{R}} \simeq 500$, nearly 1 of every 3 events would be attributable to the background. As the curves for larger $x$ suggest, cuts in $Q^{2}$ could be very helpful to suppress the background for higher $M_{\mathrm{R}}$.

Given that the structure functions will be rather well known so that these cross sections may be calculated with considerable confidence, it is natural to ask whether the anticipated experimental accuracy would permit one to subtract this background and still isolate the contribution due to a hypothetical right-handed current. Because the maximum polarization $P$ expected is about $80 \%$, one cannot simply make observations with purely right-helicity electrons but one may compare event rates seen when $P_{\mathrm{R}}=80 \%$ with $P_{\mathrm{L}}=80 \%$ (or with an unpolarized sample.) With an accumulated luminosity $L$, divided in the ratio $\sqrt{P /(1-P)}$ between 


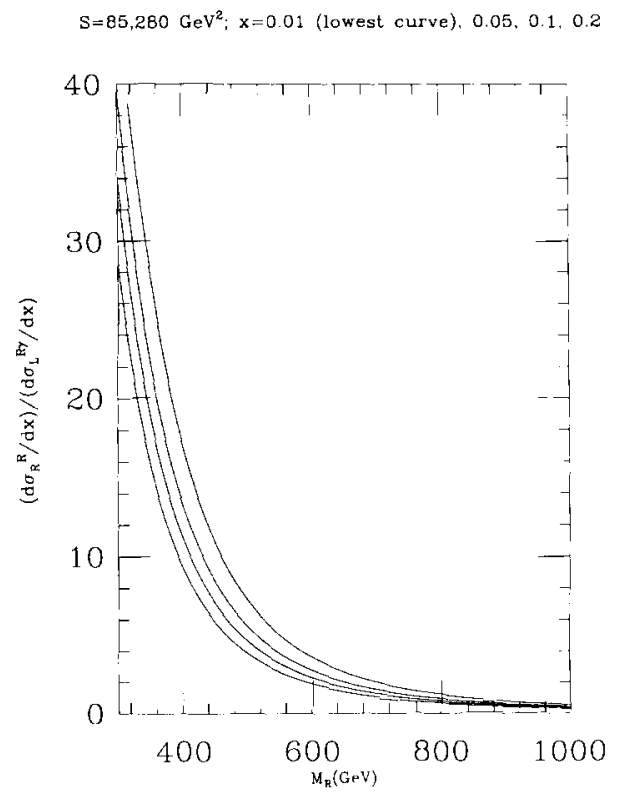

Fig. 3. The ratio $\left(\mathrm{d} \sigma_{\mathrm{R}}^{\mathrm{R}} / \mathrm{d} x\right) /\left(\mathrm{d} \sigma_{\mathrm{L}}^{\mathrm{R}, \gamma} / \mathrm{d} x\right)$ as a function of $M_{\mathrm{R}}$ for $x=0.01,0.05,0.10$ and 0.20 going from lowest to highest curve.

right-polarized and left-polarized beams, the statistical error on the ratio $\sigma^{\mathrm{R}} / \sigma^{\mathrm{L}}$ for the interaction cross section of $\mathrm{e}_{\mathrm{R}}^{-}$to that of $\mathrm{e}_{\mathrm{L}}^{-}$would be *

$$
\delta=\frac{\sqrt{P(1}-P)(\sqrt{P}+\sqrt{1-P})}{(2 P-1) \sqrt{L \sigma^{\mathrm{L}}}} .
$$

It has been claimed [5] that one might be able to observe a $3 \delta$ effect for $M_{\mathrm{R}} \simeq 300$ $\mathrm{GeV}$. Even this seems to us certainly in the range difficult to achieve with luminosities no larger than designed, and below this mass, the radiative background, even if present, is unimportant.

\section{Conclusion}

In this paper, we have performed a standard calculation of a radiative background to the search for right-handed currents. This background consists of a

* We include this here, since, as we understand them, previous estimates [1] of sensitivity have been based assuming equal running times with left- and right-polarized beams. It is intuitively clear that if $P=1$, you would perform this test solely by running with a purely right-polarized beam. For $P=80 \%$, the maximum statistical sensitivity is obtained, however, running twice as long with $P_{\mathrm{R}}=80 \%$ as with $P_{\mathrm{L}}=80 \%$, and $\delta=0.89 / \sqrt{L \sigma^{\mathrm{L}}}$. This is recommended if doing so would not introduce countervailing systematic errors and undeniable cries for more left electrons for other charged current experiments. 
helicity-flip cross section that survives in the limit $m_{\mathrm{e}} \rightarrow 0$ due to a collinear mass singularity. As is well known [11], the massless limit of processes containing mass singularities may be intimately connected with the notion of physical degeneracy and the necessity to include, to a certain order, extra diagrams corresponding to initial- and final-state degeneracy. The reader may wonder whether and to what extent these diagrams are relevant to the standard (non-degenerate) calculation presented here, given that this calculation provides a nonzero result precisely because of a mass singularity. A definite answer to this and related questions and a justification of the correctness of the standard approach in this case have been given recently [12]. Suffice it here to say that at HERA, the initial-state degeneracy, which is physically associated with the angular uncertainty of the electron beam, is much smaller that the actual mass fraction $m_{\mathrm{e}} / E_{\mathrm{c}}$ of the electron. Hence the standard calculation presented here is indeed correct.

Since this background is observable, it may be worth asking whether there might not be other reactions where a similar problem might arise. We have not completed a thorough survey of all applications of this mechanism. For the case of neutral currents at HERA, $\mathrm{e}^{ \pm} \mathrm{p} \rightarrow \mathrm{e}^{ \pm} X$, it is possible to discriminate against hard bremsstrahlung because both the quark jet and the final state electron momentum are detected. The kinematics of the non-radiative process is therefore overdetermined, and, because of this redundancy, the bremsstrahlung of a hard photon can be vetoed. The same sort of effect has been noted in $\mathrm{e}^{-} \mathrm{e}^{+}$annihilation [13], but the polarization effect is already small and an order $\alpha$ correction thereto is about 1000 times smaller *. However, off the Z-resonance, it could become more worrisome.

We would like to thank J.H. Kühn for calling our attention to ref. [13]. One of us (M. B. E.) would like to thank D.R.T. Jones for discussions and for his initial collaboration on the subject of the massless limit. The authors would like to thank J. Soffer for his hospitality at the CPT, Marseille, where portions of this work were carried out.

\section{References}

[1] Proc. Workshop Experimentation at HERA, NIKHEF, Amsterdam, June 9-11, 1983, DESY HERA 83/20 (October, 1983)

[2] R.D. Peccei, ed., Proc. HERA Workshop, Hamburg, Germany, Oct. 12-14, 1987 (DESY, Hamburg, 1988)

[3] T.D. Lee and M. Nauenberg, Phys. Rev. 133 (1964) B1549

[4] P. Langacker, in Proc. 1985 Int. Symp. on Lepton and photon interactions, ed., M. Konuma and K. Takahashi, (Kyoto U., 1986) p. 186

* As a result of a typographical error, eq. (22) of ref. [13] omits the factor $1 / z^{\prime}$ of our eq. (3.5) (J.H. Kühn, private communication). 
[5] E. Longo, in ref. [1]

[6] G. Wolf, lectures at Advanced Study Institute on Techniques and concepts of high energy physics, 1986, St. Croix, DESY 86-089, (August, 1986)

[7] R. Rückl, talk at ECFA-Workshop LEP 200, Aachen, 1986, DESY 87-021 (March, 1987)

[8] G. Altarelli and G. Parisi, Nucl. Phys. B126 (1977) 298;

G. Altarelli, Phys. Reports 81 (1982) 1

[9] B. DeWit and J. Smith, Field theory in particle physics (North Holland, Amsterdam, 1986)

[10] A.J. Buras and K.J.F. Gaemers, Nucl. Phys. B132 (1978) 249

[11] F. Bloch and A. Nordsieck, Phys. Rev. 52 (1937) 54;

T. Kinoshita, J. Math. Phys. 3 (1962) 650;

T.D. Lee and M. Nauenberg, Phys. Rev. 133 (1964) B1549

[12] H.F. Contopanagos and M.B. Einhorn, Phys. Rev. D45 (1992) 1291, 1322

[13] S. Jadach et al., Z. Phys. C38 (1988) 609 